In Cres. Vol. $4 N^{\circ} 1:$ pp. 23-32, 2013

\title{
METODOLOGÍA PARA LA DETERMINACIÓN DEL COSTO POR ESTUDIANTE DE CONTABILIDAD EN LA MODALIDAD PRESENCIAL Y SU INCIDENCIA EN LAS FINANZAS DE LA ULADECH CATÓLICA*
}

\author{
METHODOLOGY FOR THE DETERMINATION OF \\ COST ACCOUNTING STUDENT ON MODALITY AND \\ ITS IMPACT ON THE CATHOLIC ULADECH FINANCES
}

\section{Yuri Gagarín Gonzales Rentería}

\begin{abstract}
RESUMEN
El propósito de este estudio fue establecer y determinar los inductores que deben ser considerados en la metodología para acumular, sistemática e históricamente, los importes de la ULADECH Católica en el proceso de enseñanza a un estudiante de Contabilidad en la modalidad presencial, para conocer el costo razonable de profesionalizar a un estudiante. La hipótesis afirmó que la falta de aplicación de una metodología en la determinación del costo por estudiante de la Escuela Profesional de Contabilidad, en la modalidad presencial, incide en las finanzas de la ULADECH Católica. El resultado presentó una estructura de costos basada en la determinación del material directo y suministros o ambos, la mano de obra y otros costos del servicio que provee la institución durante un semestre académico. Al conocer el costo de enseñanza; la programación de becas y de estímulos fueron razonables y coherentes a la realidad de las finanzas, el valor de las pensiones permitieron promocionar la carrera. La finalidad de este estudio se podría extender a las demás carreras profesionales, para conocer los remanentes económicos del servicio de educación y sus consecuentes decisiones de no endeudamiento externo y de reinversiones, en términos de tecnología y de infraestructura, así como de capacitación a docentes, como incidencia en las finanzas.
\end{abstract}

PALABRAS CLAVE: Elementos del costo educativo, incidencia financiera, inductor, semestre académico.

* Recibido: 30 de marzo del 2013; aprobado: 28 de abril del 2013.

1 Contador Público Colegiado Certificado. Magister en Administración. Doctor en Contabilidad y Finanzas. Coordinador de Planificación de la Escuela Profesional de Contabilidad. Docente tutor investigador de la ULADECH Católica. 


\begin{abstract}
The goal of this study was to establish and determine the inductors to be considered in the methodology to build, systematic and historically, the amounts incurred by the Catholic ULADECH in the process of a student teaching of Accounting in the present modality, allowing to know the cost professionalize reasonable a student, the research hypothesis that the lack of application of a methodology in determining the cost per student at the Professional School of Accounting at the modality, affects the finances of the Catholic ULADECH. The result presents a cost structure determination based on direct material and / or supplies, labor and other service costs incurred by the institution during an academic semester. By knowing the cost of instruction, programming and stimulus grants shall be reasonable and consistent with the reality of finance, the value of pensions to promote the race. The purpose of the study is extended to other careers and let you know the economic remnants of the education service and the resulting decisions not external borrowing and reinvestment in terms of technology and infrastructure as well as training for teachers, and impact on finances. The material used is what the documentation generated in the areas of purchasing, accounting and budget. The method has been the analytic-synthetic.
\end{abstract}

KEY WORDS: Educational cost elements, financial impact, inductor, academic semester.

\title{
INTRODUCCIÓN
}

Las universidades son unidades educativas de formación profesional, personal y científica; están integradas por profesores, estudiantes y graduados. Se dedican al estudio, la investigación, la educación y difusión del saber y la cultura, y a su extensión y proyección sociales. Tienen autonomía académica, normativa y administrativa dentro de la ley Universitaria $\mathrm{N}^{\circ} 23733$.

El excedente que pudiera resultar al término de un ejercicio presupuestal anual, tratándose de universidades privadas, lo invierten a favor de la institución y en becas para estudios. No puede ser distribuido entre sus miembros ni utilizado por ellos, directa ni indirectamente.

Hasta hace unos años, las formas tradicionales de financiamiento de la educación superior en el mundo se basaban en asignaciones históricas e incrementales, que no obligaban ni llevaban a conocer de costos. Los análisis de costos surgieron en las últimas dos décadas, cuando se generaron los procesos de cambio en el financiamiento de la educación superior (Lewis, 2011).

En los inicios de 1990, el aumento en los precios de las matrículas y la reducción de los montos asignados por estudiantes llevaron a que los sistemas de administración de la educación superior en EEUU y Gran Bretaña se comenzaran a preguntar: ¿Estamos costeando?, ¿Estamos costeando de la 
misma manera?, ¿Cómo estamos costeando?, ¿Qué estamos costeando? La respuesta general fue en la educación superior no le damos importancia a los análisis de costos.

El análisis de costo puede ser expresado como costo total o expresado en términos de unidades de servicio, como: costo por estudiante, costo por docente, costo por hora crédito, entre otros.

Para cada atención y su correspondiente adquisición de bienes y servicios que realiza la Universidad Católica Los Ángeles de Chimbote (ULADECH Católica), el área de compras, logística y de contabilidad no determina ni asigna a qué escuela profesional le corresponde el costo o gasto en el que incurre, debido a que la institución no tiene establecido una metodología que permita la determinación del costo del estudiante de Contabilidad, en la modalidad presencial; lo que incide dentro de sus finanzas, puesto que no se conoce el costo por cuota de enseñanza que la ULADECH Católica invierte por cada estudiante de Contabilidad, en la modalidad presencial. Tal situación influye en el hecho de no poder proyectar sus próximas o más cercanas inversiones o reinversiones de carácter académico ni tomar decisiones acorde con la realidad académica de sus escuelas profesionales.

$\mathrm{Al}$ no contar con la metodología a proponer, la Institución objeto de estudio no costea razonablemente las salidas de recursos, la utilización de los mismos y, por tanto, los resultados económicos financieros (sus finanzas) se ven incididas.

\section{PROBLEMA}

La Universidad Católica Los Ángeles de Chimbote - ULADECH Católica no cuenta con una metodología para la determinación del costo por estudiante de la Escuela Profesional de Contabilidad en la modalidad presencial, hecho que incide en sus finanzas.

\section{OBJETIVO GENERAL}

Describir y determinar los inductores que serán empleados en la metodología para la determinación del costo por estudiante de la Escuela Profesional de Contabilidad, en la modalidad presencial, y su incidencia en las finanzas de la ULADECH Católica.

\section{OBJETIVOS ESPECÍ́FICOS}

1. Conocer la documentación que se genera en las áreas de compras, de contabilidad y de presupuesto. 
2. Describir los conceptos conocidos como inductores en los que incurre las áreas involucradas.

3. Determinar los inductores a ser incluidos en la metodología para la determinación del costo por estudiante.

\section{HIPÓTESIS}

La aplicación de una metodología en la determinación del costo por estudiante de la Escuela Profesional de Contabilidad en la modalidad presencial, incide en las finanzas de la Uladech Católica.

\section{JUSTIFICACIÓN}

En el área de Finanzas (que incluye: Compras, Contabilidad y Presupuesto), no existía metodología ni coherencia en la determinación del costo por estudiante y, por ende, dicho desconocimiento es un factor influyente en las finanzas, puesto que cuando se toman decisiones sobre inversiones, reinversiones, aumentos de pensiones, otorgamiento de becas, etc, éstas se manifiestan por el remanente en general, si lo hubiera; pero, sería interesante que las autoridades conozcan la realidad financiera de cada escuela (desde su estructura de costos unitarios, sus centros de costos hasta la determinación de los resultados) para socializar a quienes necesitan de dicha información.

\section{MATERIAL Y MÉTODOS}

El material de estudio estuvo conformado por la documentación que se generaba en las áreas de: logística, presupuesto y contabilidad; así como en la división Financiera.

La población estuvo compuesta por el universo de las áreas involucradas en la división financiera de la ULADECH Católica; y la muestra por las áreas de logística, presupuesto y contabilidad.

MÉTODOS Y TÉCNICAS

Métodos. Se consideró:

- Inductivo-deductivo, para encontrar simultaneidad con el objeto de estudio.

- Analítico-sintético, a fin de dotar el trabajo de información e interpretar los efectos en estudio y sintetizarlos en los resultados. 
- Descriptivo. El objeto de estudio fue de carácter descriptivo, para encontrar los alcances y objetivos necesarios.

- Cuantitativo, por cuanto a la metodología planteada fue necesario la determinación en unidades monetarias, el costo por estudiante.

Técnicas

- Investigación documentaria (textos, comprobantes de pago, guías, boletas, contratos etc.).

- Entrevistas a autoridades y funcionarios de la ULADECH Católica sobre las asignaciones de costos y gastos a las distintas carreras profesionales, dentro de ellas, a la carrera profesional de Contabilidad.

\section{RESULTADOS}

DETERMINACIÓN DE LA METODOLOGÍA - LOS INDUCTORES

Primero, se eligió un semestre académico, de diecisiete (17) semanas. La primera semana incluyó el proceso de matrícula, tiempo del cual se obtuvo los inductores y conceptos o ambos, que generan erogaciones y que posteriormente se clasificaron en costos y gastos.

Dentro de estos inductores encontramos:

- Sueldo y leyes sociales del Director de Escuela, del Jefe de Departamento y de la Secretaria de SUA.

- Servicios públicos de luz, agua, telefonía fija, telefonía celular, vigilancia, limpieza y mantenimiento.

Posteriormente, tomamos en cuenta el desarrollo de quince (15) semanas académicas efectivas de desarrollo molecular del Sílabo Plan de Aprendizaje.

En estas 15 semanas se encontró:

- Sueldo y leyes sociales del Director de Escuela, del Jefe de Departamento y de la Secretaria de SUA.

- Servicios públicos de luz, agua, telefonía fija, telefonía celular, vigilancia, limpieza, mantenimiento y reparación.

Además:

- Remuneración por la cantidad de horas programadas para el semestre (15 semanas) y sus respectivas leyes sociales.

- Suministros (plumones, motas, etc.). 
- Depreciación del inmueble, maquinaria y equipo (activo inmovilizado) del lugar, donde se desarrollan las clases presenciales, en la ciudad de Chimbote.

Adicionalmente:

- Prorrateo del costo de biblioteca;

- Desgaste de libros de biblioteca;

- Prorrateo de costos y gastos de decanato y rectorado. Según política.

Finalmente, se tomó en cuenta la última semana del semestre académico, que corresponde a semana de aplazados, para determinar los siguientes inductores:

- Sueldo y leyes sociales del Director de Escuela, del Jefe de Departamento y de la Secretaria de SUA;

- Servicios públicos de luz, agua, telefonía fija, telefonía celular, vigilancia, limpieza, mantenimiento y reparación.

Sobre remuneraciones:

- Remuneración por la cantidad de horas programadas para los exámenes de aplazados y sus respectivas leyes sociales.

En cuanto a los activos inmovilizados, depreciación del inmueble, maquinaria y equipo (activo inmovilizado) donde se desarrolla la evaluación de los aplazados, en la ciudad de Chimbote.

La adquisición inicial de suministros fue referencial al histórico del último semestre académico culminado, deducidos los saldos en stock no utilizados por materiales representados por plumones, motas y otros.

Por personal (mano de obra), representados por sueldos, leyes sociales y otras cargas a docentes.

Por gastos generales, representado por consumo de energía eléctrica, depreciaciones, otras remuneraciones, telefonía, mantenimiento, vigilancia, etc.

Finalmente se elabora lo siguiente: 
HOJA DE DETERMINACIÓN DEL COSTO POR ESTUDIANTE DE CONTABILIDAD EN UN SEMESTRE ACADÉMICO

\begin{tabular}{|l|l|}
\hline Concepto/inductores & Total \\
\hline $\begin{array}{l}\text { Material directo } \\
\text { Suministros. } \\
\text { Plumones. } \\
\text { Motas - otros. }\end{array}$ & \\
\hline $\begin{array}{l}\text { Mano de obra } \\
\text { Costo de hora por semestre -según cálculos. }\end{array}$ & \\
\hline $\begin{array}{l}\text { Otros costos del servicio } \\
\text { Sueldos y leyes sociales administrativos. } \\
\text { Consumo de luz. } \\
\text { Consumo de agua. } \\
\text { Consumo de telefonía fija. } \\
\text { Consumo de telefonía celular. } \\
\text { Depreciación de local. } \\
\text { Depreciación de equipos de cómputo. } \\
\text { Limpieza. } \\
\text { Mantenimiento. } \\
\text { Reparación. } \\
\text { Vigilancia - otros. }\end{array}$ & \\
\hline $\begin{array}{l}\text { Costo total } \\
\text { Número de estudiantes matriculados en el semestre académico }\end{array}$ & \\
\hline Costo unitario por cada estudiante de contabilidad & \\
\hline
\end{tabular}




\section{DISCUSIÓN}

$\mathrm{Al}$ aplicar la metodología propuesta, se determinó fehacientemente el costo unitario por estudiante de Contabilidad en la modalidad presencial, el mismo que, a diferencia de la acumulación tradicional de conceptos directos e indirectos que aplica actualmente la ULADECH Católica, permite identificar los inductores necesarios, que directamente inciden en los recursos financieros de la institución.

La determinación del costo responde a ¿qué estamos costeando?, ¿cómo estamos costeando? y ¿para qué estamos costeando? Interrogantes que, dada su vinculación con las finanzas de la institución, permiten conocer oportunamente los conceptos y recursos que se emplean, a fin de tomar las decisiones para ampliar o no la base de estudiantes y la infraestructura a demandar para el proceso de profesionalización.

Los actuales procedimientos para asignar el número de becas, los estímulos y los demás costos del servicio educativo son validados con la aplicación de la metodología propuesta para la determinación del costo. Ello permite que las asignaciones de beneficios o estímulos sean razonables en números y en incidencias financieras dentro de la institución.

Los remanentes que reportan los estados financieros no permiten identificar la procedencia, por el desconocimiento de la veracidad del costo por estudiante de las carreras profesionales; circunstancia que será corregida con los resultados de la aplicación de la metodología, puesto que todos los inductores serán cargados íntegramente a la carrera profesional, por la cual se genera, y de manera automática.

\section{CONCLUSIONES}

La aplicación de la Metodología para la Determinación del Costo por Estudiante de Contabilidad en la Modalidad Presencial y su Incidencia en las Finanzas de la ULADECH Católica permite conocer, razonablemente, el costo por estudiante y, por tanto, permite inferir en la toma de decisiones financieras, con respecto a:

- Valuación razonable de las pensiones en las carreras universitarias.

- Inversiones y reinversiones.

- Asignación de becas.

- Asignación de estímulos.

- Recategorizaciones.

- Renuncia a la exoneración de impuestos. 
- Mayor posicionamiento institucional.

- Calidad universitaria.

- Acreditación universitaria.

En cuanto al impacto, éste será derivado a:

- Local: logra la inclusión educativa por costos accesibles.

- Regional: permite profesionalizar a personas con carreras universitarias de calidad y acreditadas.

- Nacional: contribuye al desarrollo económico del país.

Las decisiones financieras en la ULADECH Católica son estimuladas por los resultados razonables de los costos educativos en que se incurren, para el proceso de enseñanza, permitiendo a la alta dirección conocer y actuar sobre los programas de estímulos y beneficios, así como la razonabilidad en el costo de las pensiones y, por ende, conocer los remanentes que permitan nuevas reinversiones tanto en infraestructura como en tecnología y capacitación.

\section{AGRADECIMIENTOS}

Muy en especial, al Ing. Dr. Julio Benjamín Domínguez Granda, por la viabilidad y la autorización en la realización de la presente investigación en la Universidad Católica los Ángeles de Chimbote

Al Lic. Felipe Carpio Vargas, por las facilidades en las entrevistas de orientación para la determinación de los inductores como base para la metodología propuesta.

\section{BIBLIOGRAFÍA CONSULTADA}

ApodACA, P. y GallarretA, L. (1999). "Propuesta de diversos indicadores del acceso/demanda de estudios universitarios". En: Indicadores en la universidad, información y decisiones. MEC/ Consejo de Universidades. Fareso SA, Madrid.

Charlton, C. (1993). La evaluación institucional en Gran Bretaña. Manuscrito no publicado. Presentación en el seminario: La evaluación universitaria. Montevideo, s.d..

CHOY, E. (2006). Alternativa Financiera - "El Costo Beneficio de la Educación Superior”. USMP.

De Miguel, M. (1999). "La evaluación de la enseñanza. Propuesta de indicadores para las titulaciones". En: Indicadores en la universidad, información y decisiones. MEC/Consejo de universidades. Fareso SA, Madrid.

GINES MORA, J. (1999). "Indicadores y decisiones en las universidades”. En: Indicadores en la universidad, información y decisiones. MEC/Consejo de universidades. Fareso SA, Madrid.

GINESTAR, A. (1990). Costos educacionales para la gerencia universitaria. EDIUNC. Argentina. 
GRAO, J. Y WINTER, R. (1999). “Indicadores para la calidad y calidad de los indicadores”. En: Indicadores en la universidad, información y decisiones. MEC/ Consejo de universidades. Fareso SA, Madrid.

LEWIS. (2011). Ministerio de Educación y Cultura de Uruguay. Obtenido http://baseddp.mec.gub.uy/ Documentos/Bibliodigi/Documentos \% 20de \% 20Trabajo \%20del\%20Rectorado \% 2011. \%20 Estudio \%20sobre \% 20indicadores \%20y \%20 costos \%20en \% 20la\%20 educacion\%20superior.pdf

Malo Alvarez, S. (1998). Los indicadores en la evaluación de la educación superior. Un recurso para la toma de decisiones y la promoción de la calidad. UNAM. México.

MARTINEZ SANDRES, F. (1999). Sistemas de información y de evaluación universitaria. Aplicación y contribución en materia de educación superior. FCU-CSIC.

PEREZ Lindo, A. (1990). "Evaluación del rendimiento de las universidades”. En: Propuesta educativa.

Petrei, H. (1989). "Costos de la educación universitaria en la Argentina”. En: Ensayos en economía de la educación. Buenos Aires.

RODRIGUEZ ESPINAR, S. (1999). "Información cualitativa y cuantitativa en el Plan Nacional de Evaluación”. En: Indicadores en la universidad, información y decisiones. MEC/Consejo de universidades. Fareso SA, Madrid.

RuIz, R. (1999). "Evaluación académica y educación superior”. En: Evaluar para transformar. IESALC UNESCO.

VIDAL, J. (1999). "Indicadores de rendimiento para las universidades españolas: necesidad y disponibilidad". En: Indicadores en la universidad, información y decisiones. MEC/ Consejo de Universidades. Fareso SA, Madrid.

ViLlarReAL, M. (1999). "La utilización de los indicadores de rendimiento en la financiación de la educación superior". En: Indicadores en la universidad, información y decisiones. Fareso SA, Madrid. 